\title{
MINIMIZED Design for the Packaging of Shenyang Specialties Against the Internet-Based Background
}

\author{
YANG Meng $^{1, a}$, ZHANG Xiaohui $^{1}$ \\ ${ }^{1}$ Shenyang Aerospace University School of Design \& Art, 110136 Shenyang, China
}

\begin{abstract}
The focus herein is innovative design ideas for the packaging of Shenyang specialties under the concept of minimization. Through analyzing the current status of the packaging of Shenyang specialties, the relevance between minimized design and specialty package is discussed, while based on actual cases, studies are carried out to find solutions for problems including waste of resources resulted from excessive packaging, homogenization of package images, blurring of audience identification, and unitary sales channel. By redesigning the packaging of Shenyang specialties against the internet-based background and increasing visibility of specialty products, new ideas and methods for the package design of Shenyang specialties are provided taking minimized concept as the entry point, thus to realize value guidance and culture communication under the concept of minimization.
\end{abstract}

\section{Introduction}

The focus herein is innovative design ideas for the packaging of Shenyang specialties under the concept of minimization. Through analyzing the current status of the packaging of Shenyang specialties, the relevance between minimized design and specialty package is discussed, while based on actual cases, studies are carried out to find solutions for problems including waste of resources resulted from excessive packaging, homogenization of package images, blurring of audience identification, and unitary sales channel. By redesigning the packaging of Shenyang specialties against the internet-based background and increasing visibility of specialty products, new ideas and methods for the package design of Shenyang specialties are provided taking minimized concept as the entry point, thus to realize value guidance and culture communication under the concept of minimization.

\section{The current situation and problems of the packaging of Shenyang specialties}

Shenyang is a city with distinct regional features. Since specialties are an important driving force for the development of tourism economy, it's an important task of package design to endow specialties with vitality. To make full use of e-commerce platforms to expand the market and achieve trans-regional and sales, the packaging of specialties must be adjusted and modified to build the brand image of Shenyang specialties and improve the cultural connotation and artistic quality of package design, and thus to satisfy requirements on packaging brought by e-commerce platforms. Main problems existing in the packaging of Shenyang specialties:

\subsection{Problems during the process of purchasing}

Research shows that, there are mainly two problems existing in the packaging of Shenyang specialties during the process of purchasing: the first is that the awareness of brand promotion is poor. With respect to extraction and use of local elements, the commonly applied method is to pile up some simple visual elements of products, which is not sufficient to demonstrate the relationship between elements and the package, nor the unique regional and ethnical appeals. Such brand images are not beneficial for communication and promotion. The second is that the styles are highly homogenized. Style designs mainly use regular rectangular boxes, which lacks creativity and won't arouse consumers' interests in buying or desire to consume.

\subsection{Problems after purchasing}

After purchasing behavior is completed, the other identity of commodity package emerges, namely, it should be environmental friendly, without excessive packaging or waste producing. Results of excessive packaging: the first is increasing pressure on logistics and transportation. This is reflected in aspects including excessively bulky and heavy packaging, and poorly arranged internal structure, and too many materials used, all of which are the results of exquisite packaging. Consequently, the packaging is beyond general requirements for commodity packing in terms of size, space and volume, and brings heavy burden to logistics. The second is lacking of considerations for the

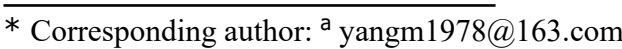


sustainable use of materials. Selection of packaging materials lacks environmental considerations, thus materials that are difficult to recycle including plastics and paper are used most commonly, which will cause environmental pollution and waste of resources.

\section{Analysis on demands for minimized packaging design for Shenyang specialties against the internet-based background}

\subsection{Analysis on merchants' demand for minimized packaging of specialties}

Compared to the business model of physical stores, online sales can save large quantities of promotional costs and store-related costs, as all the promotions can be finished through online platforms. Under this new selling model, commodities can be identified through code scanning, and information about them will be clear at a glance, hence, the target of this event will be achieved as long as the exclusive express packaging can communicate information $^{[1]}$. To continuously expand the market share, merchants must increase the visibility of commodities, and enable the packaging to win a place in fierce market competition and draw people's attention, thus to bring consumers with visual enjoyment and spiritual relax. While designing the packaging of Shenyang specialties, more attention should be paid on the visual image creation for the packaging, to allow consumers to see the appeal of Shenyang specialties and stimulate their design to buy.

\subsection{Analysis on consumers' demand for minimized packaging of specialties}

As participants of the shopping process, consumers' demand for packaging usually determines the development trend of packaging. Consumers wish to receive commodities with express packaging that is flawless, easy to open and humanized, and at the same time, has certain regional characters to highlight unique historical and cultural information of the place of origin. Therefore, the selection of packaging materials is very important. Research shows that, people prefer environmental friendly materials with resources from the nature, which bring people natural senses of intimacy, reality and plainness, and satisfy their emotional demands.

Using natural materials with regional characters can not only demonstrate the unique cultural appeal, but also realize the environmental friendly design concept. While handling materials, the original mechanism of natural materials should be made good use of, in order to minimize machining and retain the beauty of the materials themselves. Consumers who buy specialties prefer the primitive feeling brought by natural materials. For example, using straws to create plain packaging for Shenyang specialties and using natural materials to make packaging containers will touch the souls of consumers, without losing regional characters of Shenyang.

\subsection{Analysis on demand of logistics for minimized packaging of specialties}

As a means of goods transportation, logistics is the bridge for merchants to deliver goods to consumers, and plays an important role in the process of online shopping ${ }^{[2]}$. For commodities bought online, the packaging structure should be optimized not only to satisfy functions including protecting commodities, facilitating transportation and being durable, but also to lower the production cost and protect the environment by using least possible materials, without reducing functions of commodity packaging. The minimized design concept should be promoted, by reasonably saving materials used in packaging and giving full play to recycling of resources.

Compared to static conditions, commodities are more likely to be damaged during the process of transportation because they are vulnerable to impact of external forces and tossing resulted from road transportation. With respect to this problem, the most common solution is to add a larger external package filled with corrugated paper, white foam filler and dunnage bags as buffer materials. Therefore, appropriate structures should be selected based on the characteristics of commodities and materials, to provide effective protection and optimize the packaging design to be suitable for logistics handling and transportation. Internal structure of specialty packaging can be optimized based on ergonomic principles, to reduce the size and volume of packaging, use of materials, and pressure on logistics ${ }^{[3]}$.

For example: Figure 1 is the combined dried fruit package design of Taiwan good fruit that packs cantaloupe, pineapple, apple and orange separately and then together into an external package, which reduces the overall volume and further makes the packaging suitable for logistics transportation.

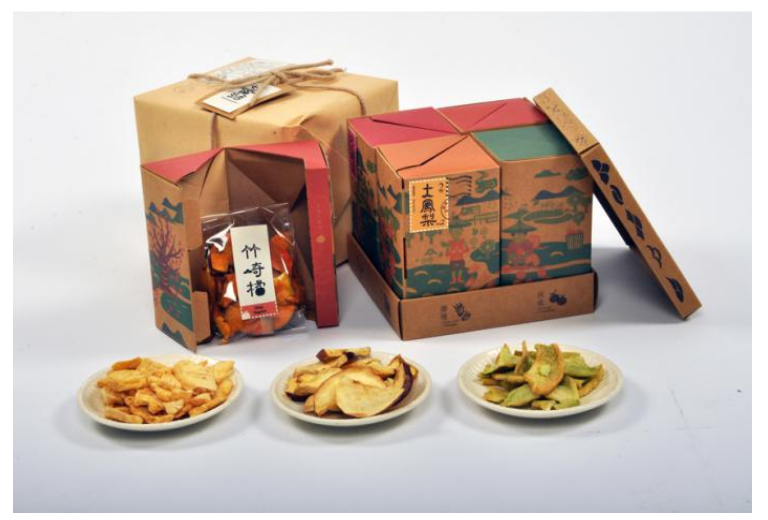

Figure 1. Combined dried fruit package of Taiwan good fruit. 


\section{Solutions to minimized design for the packaging of Shenyang specialties against the internet-based background}

\subsection{Highlighting the reasonability of packaging structure}

Packaging structure indicates the mutual constructive relationship among the shapes of different parts or units $^{[4]}$. As specialties against the internet-based background, the first thing to think about should be innovative and pioneering design of the packaging structure. Design of packaging structure should take in to account various factors including the shape characters of commodities, features of packing materials, spatial requirements of transportation and storage, and ergonomic performance when carried and used by consumers.

Commodity categories, size requirements and classification provided by e-commerce platforms are rather unitary. Therefore, to divide sizes reasonably utilizing limited space, studies and classifications of the commodity attributes should be carried out. Packaging structure and materials should be designed based on different commodity attributes, to make commodity packaging more suitable for sales on e-commerce platforms without reducing the life span of the packaging. As shown in Figure 2, the packaging of Charlie Bohea Tea boasts a design concept different from ordinary ones, which uses an interesting hexagon box that looks like a honeycomb, to distinguish itself from the general concept of demonstrating the profound history and culture of tea. Trying to break out of tradition and demonstrate the modern characteristics of tea, Charlie Bohea Tea tells consumers that this is a both traditional and modern tea through the structure and color system of the packaging.

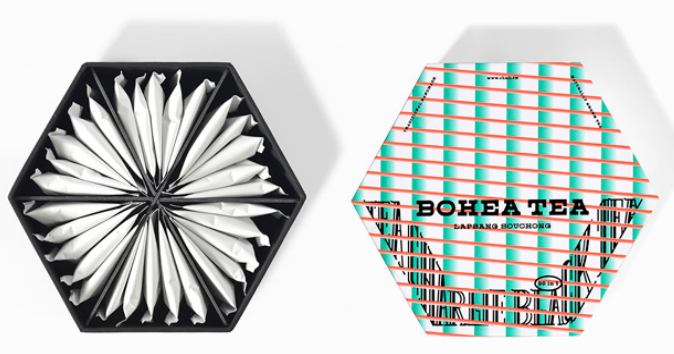

Figure 2. The packaging of Charlie Bohea Tea.

\subsection{Emphasizing standardization of packing sizes}

Confronted with the uncertainty of shopping environment against the internet-based background, the packaging design should take full account of the unification and standardization of packing sizes. This will not only protect commodities from broken during transportation, but also lower the transportation costs, thus to gain consumers' recognition of the quality and awareness and favorability of the brand.

From the perspective of functions, the packaging structure involves commodity protection, and being convenient for storage, transportation, use and sales. Convenience is an important factor for the design of packaging structure ${ }^{[5]}$, which is mainly reflected in aspects including transportation, carrying and use. During material selection and production of the packaging, attributes of specialties should be analysed , to enable the packaging to improve its protection of safety while being environmental friendly. As shown in Figure 3, TAKECO 1982 is a Japanese mixed seasoning brand, which, inspired by cargo transportation and sail elements, designed the packaging into triangles with patterns on them representing the fusion of seasoning cultures, while the size of the box is suitable for holding with two hands, and giving out as a gift. The triangle shape and appreciate size have made it more convenient for transportation of online sales.

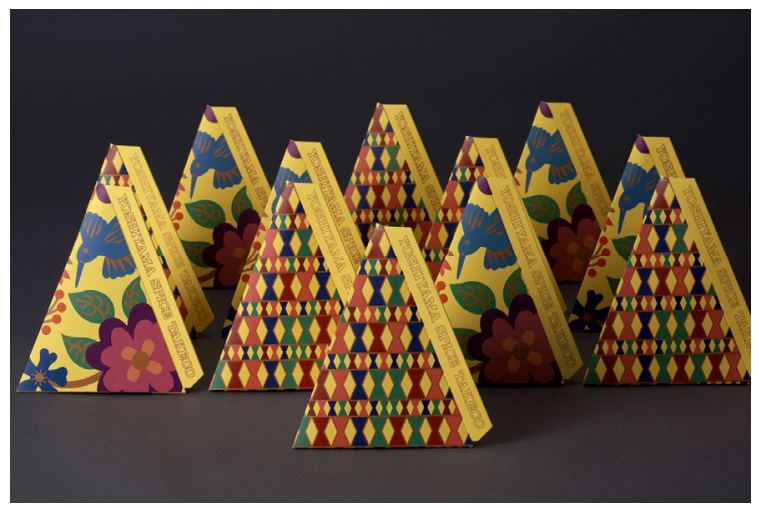

Figure 3. The packaging of Takeco Yoshiyama seasonings.

\subsection{Modularizing the configuration of packaging space}

Modularized design and configuration space inside the packaging box will not only facilitate classification, but also utilize the limited space reasonable and reduce the pressure on logistics ${ }^{[6]}$. If the space is not configured reasonably, use of fillings will be increased imperceptibly, and accidental damages to commodities are likely to happen. This will bring heavy burden to logistics and increase merchants' transportation costs. Small-sized merchants operating on e-commerce platforms often attract consumers to buy the commodities by covering the postage, hence how to save money for e-commerce buyers and sellers becomes our focus. Therefore, during this process, the packing sizes of commodities are the priority of design. For example, in Figure 4, the packaging of Shenyang agricultural specialties targeted at e-commerce platforms is designed by the author with patterns based on the perspective of Northeast folk customs, while the graphic and cultural renderings are finished with "Eight Weird of the Northeast" as the regional cultural symbol. Different 
from ordinary plastic vacuum packaging, it is designed with aluminum can as packing material to distinguish it from common rice products in bulk and in bags. This packaging not only satisfies the demand for shelf life of rice products, but also conforms to consumers' psychological expectation for the preservation effect of the product.

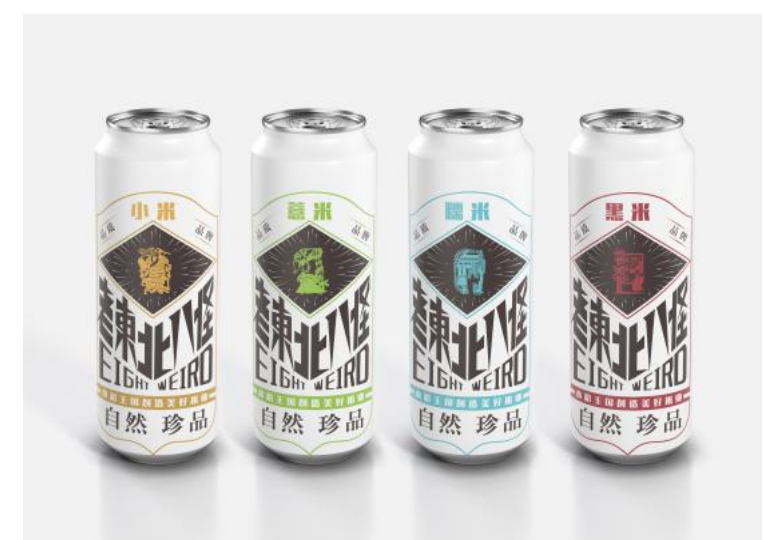

Figure 4. Author design the packaging of "Eight Weird of the Northeast" rice.

\section{Conclusion}

As the first step for consumers to learn about commodities and generate the willingness to buy, packaging is an invisible bridge between commodities and consumers. With the development of technology, packaging design of commodities is also moving towards a deeper level. Against the internet-based background, the involvement of e-commerce channels provides commodity sales with more alternatives. Based on current consuming model of e-commerce, the concept of minimized design for the packaging of specialties is put forward herein, to come up with and formulate packaging design planning that fits different ideas from the perspectives including regional culture of brand, target consumer group, sales characters and demand of times, and further to realize demand of Shenyang specialties for minimized packaging design through demonstration of localized values, recycling of materials and standardization of sizes. The packaging structure is to be optimized based on researches on consumers' psychology and ergonomics, to design packages that are suitable for the internet-based environment and satisfy consumers demands by optimizing.

\section{References:}

1. K. Bian, Y. Yang, Packaging Engineering, 33, 56 (2012)

2. H. Tian, Y. Qiu, Modern Decoration, 1, 15 (2014)

3. W. Wang, Z. Wu, Packaging Engineering, 24, 8 (2015)

4. Z. Wei, J. Wei, J. Lin, Packaging Engineering, 24, 88 (2014)

5. F. Zeng, W. Wang, F. Wang, Design, (2014)

6. H. Yang, Art Panorama, 07, 141 (2016) 